\title{
Differentiation of endogenous Erythropoietin and exogenous ESAs by Western blotting with deglycosylation
}

\section{Yukiko Yasuoka}

Kitasato University School of Medicine https://orcid.org/0000-0002-9380-1653

\section{Takashi Fukuyama}

KItasato University Medical Center https://orcid.org/0000-0003-1772-3478

\section{Yuichiro Izumi}

Kumamoto University Graduate School of Medical Sciences https://orcid.org/0000-0003-0827-9619

\section{Tetsuro Yamashita}

Iwate University https://orcid.org/0000-0002-1047-1290

\section{Yushi Nakayama}

Kumamoto University Graduate School of Medical Sciences

\section{Hideki Inoue}

Kumamoto University Graduate School of Medical Sciences

\section{Kengo Yanagita}

Kitasato University School of Allied Health Sciences

\section{Tomomi Oshima}

Kitasato University School of Medicine https://orcid.org/0000-0003-2368-716X

\section{Taiga Yamazaki}

Kitasato Univeristy Medical Center https://orcid.org/0000-0003-4674-0883

\section{Takayuki Uematsu}

Kitasato University Medical Center https://orcid.org/0000-0002-3680-7231

\section{Noritada Kobayashi}

Kitasato University Medical Center https://orcid.org/0000-0003-0321-6379

\section{Yoshitaka Shimada}

Kitasato University Medical Center

\section{Yasushi Nagaba}

Kitasato University Medical Center https://orcid.org/0000-0001-8614-319X

\section{Masashi Mukoyama}

Kumamoto Univerity Graduate School of Medical Sciences

\section{Yuichi Sato}

Kitasato University School of Allied Health Sciences

Jeff M. Sands 
Emory University School of Medicine https://orcid.org/0000-0001-9822-0607

\section{Katsumasa Kawahara}

Kitasato University School of Medicine https://orcid.org/0000-0003-4816-0321

\section{Hiroshi Nonoguchi ( $\nabla$ nono@insti.kitasato-u.ac.jp )}

Kitasato University Medical Center https://orcid.org/0000-0001-7831-7380

\section{Short Report}

Keywords: erythropoietin, erythropoiesis-stimulating agents, doping, Western blot anakysis, Liquid Chromatography/Mass Spectrometry analysis

Posted Date: July 6th, 2020

DOI: https://doi.org/10.21203/rs.3.rs-39878/v1

License: (c) (i) This work is licensed under a Creative Commons Attribution 4.0 International License. Read Full License 


\section{Abstract}

Doping tests for illegal use of erythropoiesis-stimulating agents (ESAs) have been developed. Here, we show a new Western blotting to distinguish endogenous erythropoietin (Epo, 35-38 kDa) and exogenous ESAs (epoetin alpha and beta; 38-42 kDa, darbepoetin alpha; 47-50 kDa, epoetin beta pegol; 93-110 kDa). In contrast, Liquid Chromatography/Mass Spectrometry analyzed all endogenous Epo and exogenous ESAs as delycosylated $22 \mathrm{kDa}$ Epo, indicating that LC/MS analysis could not distinguish Epo and ESAs. We believe that our Western blotting is useful to protect against illegal use of ESAs.

\section{Main Text}

Erythropoiesis stimulating agents (ESAs) have been invented to treat patients with renal anemia ${ }^{1}$. Illegal usage of ESAs by athletes has been checked using tests for doping ${ }^{2,3}$. To detect illegally used ESAs in urine, isoelectrical focusing (IEF) and/or SAR-PAGE after enrichment and purification of Epo have been recommended by the World Anti-Doping Agency (WADA ${ }^{2-7}$. Liquid Chromatography/Mass Spectrometry (LC/MS) has also been used for the detection of $E S A^{8}$. Pre-purification of Epo or loading a large amount of samples are required to observe Epo by Western blotting or SDS-PAGE with Coomassie brilliant blue staining $^{9-11}$. We have developed a new method for the detection of Epo in urine, plasma/serum and tissue by Western blotting ${ }^{12,13}$. Pre-purification of Epo is not required in our method. We cut the $22 \mathrm{kDa}$ band of deglycosylated Epo isolated from an anemic patient's urine and analyzed it by LC/MS. We found that the band contained human Epo, proving the correctness of our method ${ }^{13}$. Using this method, we compared endogenous Epo and exogenous ESAs. Recombinant human Epo was detected in a broad band at 34-43 $\mathrm{kDa}$ and endogenous Epo was detected at 35-38 kDa (Fig. 1). ESAs were detected at larger sizes: epoetin $\mathrm{a}$ and $\mathrm{b}$ at 38-42 kDa, darbepoetin a at 47-50 kDa, and epoetin b pegol at 93-110 kDa (Fig. 1). The bands of endogenous Epo and exogenous ESAs except epoetin b pegol were shifted to $22 \mathrm{kDa}$ by deglycosylation with PNGase. The band of polyethylene glycol (PEG)-bound epoetin b pegol was shifted to 81-93 kDa. LC/MS analysis detected endogenous rhEpo and exogenous ESAs both in glycosylated and deglycosylated forms as deglycosylated Epo of $22 \mathrm{kDa}$ (Suppl Table 1). We have shown that endogenous Epo is detected as a $22 \mathrm{kDa}$ band in our previous report ${ }^{13}$. Our data clearly show that LC/MS analysis cannot differentiate endogenous rhEpo and exogenous ESAs since LC/MS analysis needs trypsin digestion of samples ${ }^{14}$. Plasma Epo measurements also cannot differentiate endogenous Epo and ESAs. In contrast, Western blotting can easily distinguish endogenous Epo and exogenous ESAs. The shift of a band to $22 \mathrm{kDa}$ could confirm that the band is endogenous Epo or exogenous ESAs.

One of the advantages of degycosylation is the increase of the sensitivity of detection (Fig. 2). Higuchi, et al. showed deglycosylation of Epo using SDS-PAGE and Coomassie brilliant blue staining ${ }^{9}$. They had to load a huge amount of the samples. Epo protein expression by Western blot has been reported but prepurification of Epo from samples were required, which caused difficulty for quantitative analysis ${ }^{12,13}$. Prepurification of Epo from sample is not required in our method. The most important advantage of our method is its high sensitivity and specificity. 1-2 ng of Epo is required for the detection by LC/MS 
analysis, while only a few pg of Epo is needed for Western blotting. The differentiation of endogenous Epo and exogenous ESAs were simply performed by the differences of band sizes (Fig. 1). The shift of a band to $22 \mathrm{kDa}$ by deglycosylation confirmed the presence of some kind of Epo. The sensitivity of deglycosylated Epo and ESAs are at least 10 times higher than glycosylated endogenous Epo and ESAs. The control band of recombinant human Epo helps quantitative analysis of ESAs.

In conclusion, the highly specific and sensitive detection of endogenous Epo and exogenous ESAs became possible with our Western blotting method. The shift of bands by deglycosylation is useful to confirm that the band is Epo or ESAs. Although LC/MS analysis is useful to detect urine Epo, LC/MS cannot differentiate endogenous Epo and exogenous ESAs. We believe that our method is useful to protect against illegal use of ESAs.

\section{Methods}

\section{Materials}

Urine was collected from an anemic patient. Our protocols were checked and approved by the Ethics Committee at Kitasato University Medical Center (25-2, 2018032, 2019029). Informed consent was obtained from the patient.

\section{Western blot analysis with deglycosylation}

Western blot analysis was performed as described previously ${ }^{11,12}$. Urine samples were obtained from an anemic patient. Urine samples were concentrated by Vivaspin 20 (GE Healthcare Bio-Science AB. Sweden) and used for Western blot. Urine samples and ESAs were used directly or after deglycosylation for SDS-PAGE (10-20\% gradient gel, Cosmo Bio No. 414893, Tokyo, Japan). Recombinant human Epo (rhEpo, 587102, BioLegend, San Diego, USA) was used as a positive control. Glycosylated and deglycosylated rhEpo were combined. After SDS-PAGE, proteins were transferred to a PVDF membrane (Immobilon-P, Merck Millipore, IPVH00010) with $160 \mathrm{~mA}$ for $90 \mathrm{~min}$. The membrane was blocked with 5\% skim milk (Morinaga, Japan) for 60 min and incubated with the antibody against Epo (Santa Cruz, sc$5290,1: 500)$ for $60 \mathrm{~min}$ at room temperature. After washing, the membrane was incubated with a secondary antibody (goat anti-mouse IgG $(\mathrm{H}+\mathrm{L})$ (Jackson ImmunoResearch Laboratories, 115-035-166, 1:5,000) for 60 min. Bands were visualized by the ECL Select Western Blotting Detection System (GE Healthcare Bio-Science AB, RPN2235) and LAS 4000 (Fujifilm).

Deglycosylation was performed as described previously ${ }^{11,12}$. To $10 \mathrm{ml}$ of concentrated urine or diluted ESAs, $1 \mathrm{ml}$ of $10 \%$ SDS was added and boiled for $3 \mathrm{~min}$. Then, $11 \mathrm{ml}$ of $2 x$ stabilizing buffer was added, and $2 \mathrm{ml}$ of PBS or PNGase (Takara, 4450) was added. The samples were incubated in a water bath at $37^{\circ} \mathrm{C}$ for $15-20 \mathrm{hr}$. The $2 \mathrm{x}$ stabilizing buffer contained $62.5 \mathrm{mM}$ Tris- $\mathrm{HCl}$ (pH 8.6), $24 \mathrm{mM}$ EDTA, 2\% NP-40 and $4 \%$ 2-mercaptoethanol. Urine Epo concentrations were measured by CLEIA (SRL, Tokyo, Japan, using Access Epo by Beckman Coulter, Brea, USA). 


\section{LC/MS analysis}

The glycosylated and deglycosylated bands of rhEpo and ESAs were subjected to LC/MS (Suppl. Fig. 1). The negatively stained (293-57701, FUJIFILM Wako Pure Chemical, Osaka, Japan) Epo and ESAs bands were excised from the SDS-PAGE gel, and in-gel tryptic digestion was carried out using ProteaseMAX reagent (Promega, WI, USA) according to the manufacturer's protocol. The peptides were separated by Lcolumn2 ODS ( $3 \mathrm{~mm}, 0.1 \times 150 \mathrm{~mm}, \mathrm{CERI}$, Tokyo, Japan) at a flow rate of $500 \mathrm{nl} / \mathrm{min}$ using a linear gradient of acetonitrile (5\% to $45 \%$ ). Nano-LC-MS/MS analyses were performed with an LTQ-Orbitrap XL mass spectrometer (Thermo Fisher Scientific, MA, USA) as previously described ${ }^{14}$.

\section{Data availability}

The detailed data of LC/MS analysis are available upon request from the corresponding author $(\mathrm{HN})$ on request.

\section{Code availability}

All code used to process the data and conduct analyses described in the Methods. Gels of Western blotting were prepared using Adobe Photoshop CS6.

\section{Declarations}

\section{Funding}

This study was supported by a Grant-in Aid for Scientific Research from the Ministry of Education, Culture, Sports, Sciences and Technology of Japan (26461259(KK), 16K09654(HN), 16K19493(YI), 16K08505(YY), 17K16578(TF), 18K08247(YI) and 19K09226(TF)) and by the Science Research Promotion Fund from the Promotion and Mutual Aid Corporation for Private Schools of Japan (HN).

\section{Author contributions}

YY, TF, YI and HN designed the research; YN, HI, KY, TO, NK and HN performed deglycosylation, YY, TF, TaY, TU, and HN performed Western blot analysis; TeY performed LC/MS analysis. YoS, YN and HN collected patient's urine. MM, YuS, JMS and KK advised on the experimental design and data interpretation. YY, TF, YI, JS and HN wrote the manuscript.

\section{Competing interests}

The authors have no financial conflicts to declare.

\section{References}

1. Kalantar-Zadeh K. History of erythropoiesis-stimulating agents, the development of biosimilars, and the future of anemia treatment in nephrology. J. Nephrol.45, 235-247 (2017) 
2. WADA EPO Working Group. Harmonization of analysis and reporting of erythropoiesis stimulating agents (ESAs) by electrophoretic techniques (TD2014ZEPO) in WADA Technical Document (TD2019INDEX) (2019)

3. Reichel C. Recent developments in doping testing for erythropoietin. Bioanal. Chem.401, 463-481 (2011)

4. Heuberger JAAC, et al. Sensitivity and specificity of detection methods for erythropoietin doping in cyclists. Drug Test Anal. 11,1290-1301 (2019)

5. Lasne F, de Ceaurriz J. Recombinant erythropoietin in urine. 405, 635 (2000)

6. Lasne F, Martin L, Crepin N, de Ceaurriz J. Detection of isoelectric profiles of erythropoietin in urine: differentiation of natural and administered recombinant hormones. Anal Biochem. 311, 119-126 (2002)

7. Reichel C. SARCOSYL-PAGE: a new electrophoretic method for the separation and immunological detection of PEGylated proteins. Methods Mol Biol.869, 65-79 (2012)

8. Okano M, Sato M, Kageyama S. Identification of the long-acting erythropoiesis-stimulating agent darbepoetin alfa in human urine by liquid chromatography-tandem mass spectrometry. Anal Bioanal Chem. 406, 1317-1329 (2014)

9. Higuchi M, Oh-eda M, Kuboniwa H, Tomonoh K, Shimonaka Y, Ochi N. Role of sugar chains in the expression of the biological activity of human erythropoietin. J Biol Chem.267, 7703-7709 (1992)

10. Yamaguchi K, Akai K, Kawanishi G, Ueda M, Masuda S, Sasaki R. Effects of site-directed removal of $\mathrm{N}$-glycosylation sites in human erythropoietin on its production and biological properties. J Biol Chem.266, 20434-20439 (1991)

11. Kodama D, et al. Production of human erythropoietin by chimeric chickens. Biochem Biophys Res Commun.367, 834-839 (2008)

12. Yasuoka $Y$, et al. Fludrocortisone stimulates erythropoietin production in the intercalated cells of the collecting ducts. Biophys. Res. Commun.503, 3121-3127 (2018)

13. Yasuoka Y, F, et al. Erythropoietin production by the kidney and the liver in response to severe hypoxia evaluated by Western blotting with deglycosylation. Physiol Rep 8, e14485 (2020)

14. Takahashi D, Kawamura Y, Yamashita T, Uemura M. Detergent-resistant plasma membrane proteome in Oat and Rye: similarities ad dissimilarities between two monocotyledonous plants. Proteome Res.11,1654-1665 (2012)

\section{Supplementary Figure Legends}

\section{Suppl Fig. 1. SDS-PAGE and negative staining for LC/MS analysis.}

(left) rhEpo (19.9 ng) and ESAs )epoetin a; 7.5 IU, epoetin b; $7.5 \mathrm{IU}$, darbepoetin a; 50 ng, epoetin b pegol; $104 \mathrm{ng}$ ) were applied to SDS-PAGE and stained by negative staining. All of the glycosylated and deglycosylated bands were stained by negative staining. (right) The bands were cut and proceeded to 
LC/MS analysis. Blue arrows indicate glycosylated Epo and ESAs. Red arrows show deglycosylate Epo and ESAs.

\section{Suppl Table 1. LC/MS analysis of rhEpo and ESAs.}

The results of LC/MS analyses are shown. The numbers $\triangle-\otimes$ correspond to the numbers in right panel of Suppl Fig.1 The matched peptide with Epo was shown by bold red. LC/MS analysis showed that all of the bands contained $22 \mathrm{kDa}$ Epo.

\section{Figures}

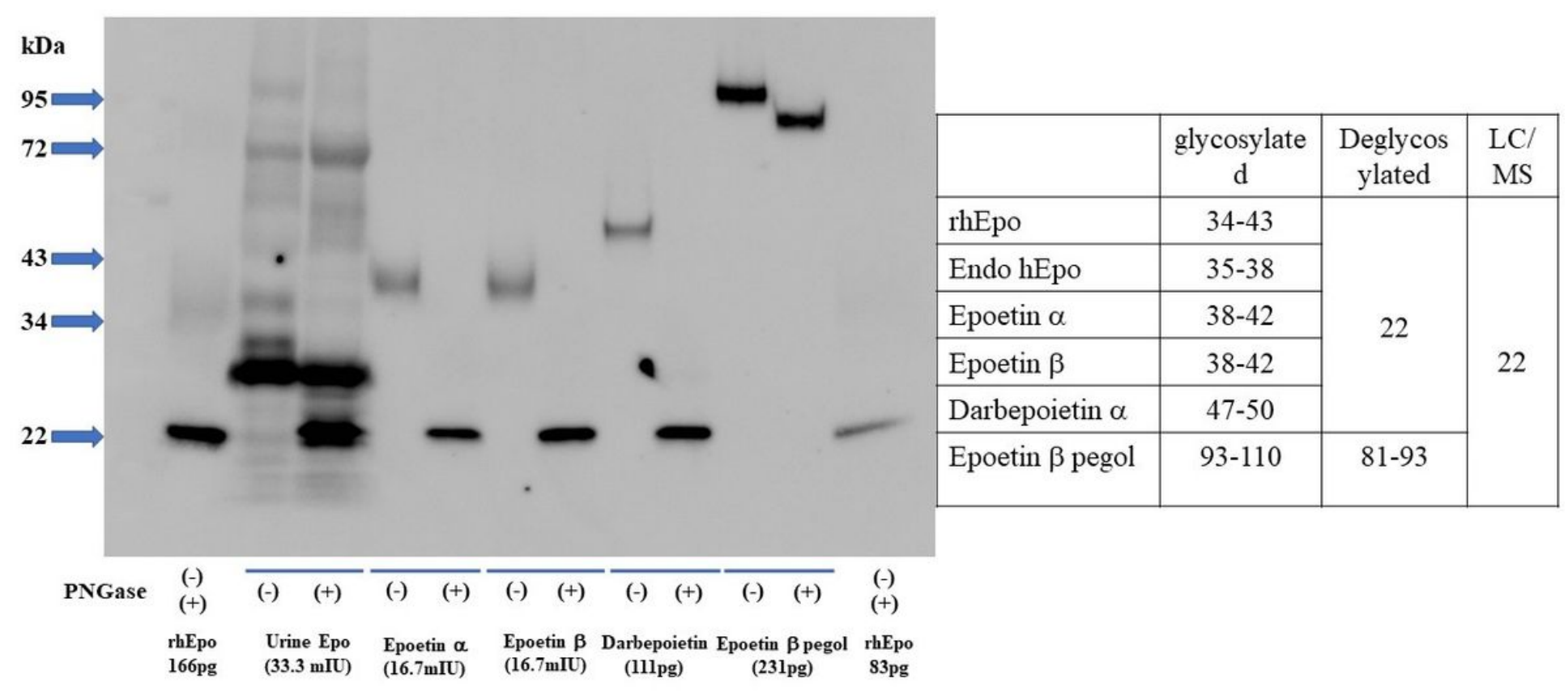

\section{Figure 1}

Detection of urinary Epo and ESAs by Western blotting with deglycosylation. Urinary Epo and ESAs were investigated by Western blotting. Endogenous urinary Epo was smaller than ESAs. The bands of endogenous Epo and ESAs except epoetin $₫$ pegol shifted to $22 \mathrm{kDa}$ by deglycosylation. The band of PEG-bound epoetin $\triangle$ pegol was slightly reduced in size by deglycosylation. The sizes of endogenous Epo and ESAs before and after deglycosylaton by Western blotting and by LC/MS analysis (Suppl Table 1) were summarized in the Table. The amount of samples are as follows: urine Epo (33.3 mIU), epoetin $\square$ and $\otimes(16.7 \mathrm{mlU})$, darbepoietin $(111 \mathrm{pg})$ and epoetin $\otimes$ pegol $231 \mathrm{pg}$. rhEpo, recombinant human EPo, Endo Epo, endogenous Epo. 


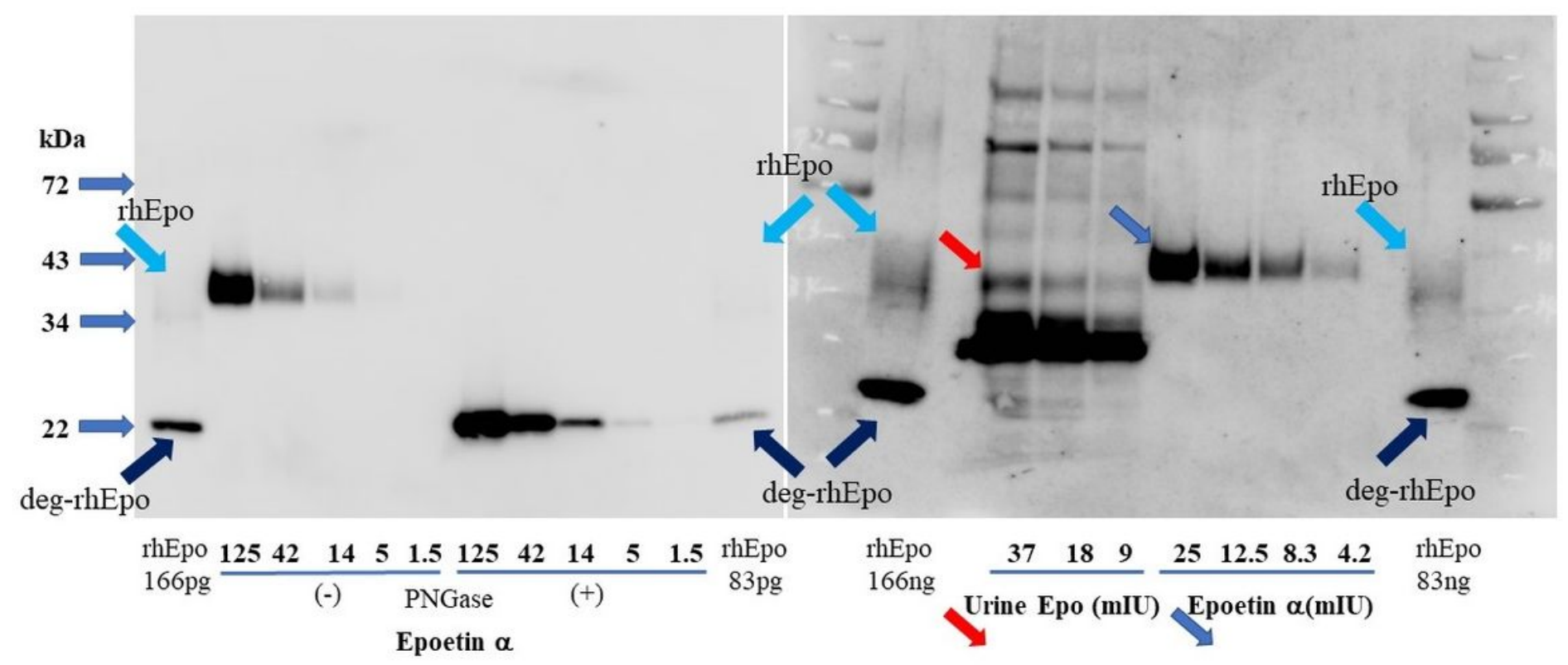

Figure 2

Detection of urine Epo and ESAs by Western blotting. (left panel) Glycosylated and deglycosylated epoetin a (38-42 and $22 \mathrm{kDa}$, respectively) was investigated by Western blotting. The detection limit was improved by deglycosylation. (right panel) Urinary Epo and epoetin $₫$ without deglycosylation were

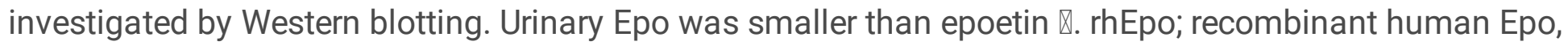
deg-rhEpo; deglycosylated recombinant human Epo

\section{Supplementary Files}

This is a list of supplementary files associated with this preprint. Click to download.

- SupplFig.1.JPG

- SupplTable1.JPG 